\title{
Eosinophilic leukemoid reaction associated with carbamazepine hypersensitivity
}

\author{
Gaurish Laad, M. F. M. Miranda \\ Department of Skin and VD, Goa Medical College and Hospitals, Bambolim Goa, India.
}

Address for correspondence: Gaurish Laad, H-3, Green Land colony, Behind Military Hospital, Panaji Goa - 403001, India. E-mail: gaurishlaad@indiatimes.com

\begin{abstract}
Carbamazepine is widely used in the treatment of epilepsy, neuralgic pain, and bipolar affective disorders. Several adverse drug reactions have been described during the course of carbamazepine administration, including exanthemata and hematological reactions. Carbamazepine is one of the common drugs that have been implicated in the etiology of drug hypersensitivity syndrome. A 50-year-old male presented with generalized erythroderma following 10 weeks of ingestion of carbamazepine $\mathbf{2 0 0} \mathbf{~ m g}$ daily for idiopathic epilepsy. His systemic examination was within normal limits. Blood counts revealed marked eosinophilia of $52 \%$ (absolute eosinophil count of 10,400 per $\mathrm{mm}^{3}$ ). Bone marrow aspiration revealed a moderate increase in the eosinophilic series with cells showing normal morphology. The eosinophil counts returned to normal after 2 weeks of oral prednisolone therapy. This case is reported because of its rarity in the Indian medical literature.
\end{abstract}

KeY WoRDs: Carbamazepine, Hypersensitivity, Eosinophilic leukemoid reaction.

\section{INTRODUCTION}

Carbamazepine is a widely used anticonvulsant which often causes adverse drug eruptions. Carbamazepine hypersensitivity reactions may mimic viral infections, glandular fever, or pseudo-lymphoma. ${ }^{[1-3]}$ Eosinophilia may occur as a part of carbamazepine hypersensitivity but leukemoid reaction secondary to carbamazepine therapy is very rare. A case of carbamazepine hypersensitivity associated with neutrophilic leukemoid reaction has been reported in literature. ${ }^{[4]}$ We report a case of carbamazepine hypersensitivity associated with eosinophilic leukemoid reaction.

\section{CASE REPORT}

A 50-year-old male presented with generalized erythema, scaling and edema of the face and extremities of eight days' duration following ten weeks of ingestion of carbamazepine $200 \mathrm{mg}$ daily for idiopathic epilepsy. After four days of commencement of the eruption, carbamazepine was stopped by a private practitioner. After another four days he came to our dermatology department. On examination, he had generalized erythroderma, and pitting edema of the face, hands and feet. He had fever $\left(40^{\circ} \mathrm{C}\right)$, but no lymphadenopathy or hepatosplenomegaly. General

How to cite this article: Laad G, Miranda MFM. Eosinophilic leukemoid reaction associated with carbamazepine hypersensitivity. Indian J Dermatol Venereol Leprol 2005;71:35-7.

Received: June, 2004. Accepted: October, 2004. Source of Support: Nil. Conflict of interest: None declared. 
examination revealed a normal pulse, blood pressure and respiratory rate. Systemic examination did not reveal any abnormalities.

Investigations on admission showed $\mathrm{Hb}$ of $13.5 \mathrm{gm} \%$, total leukocyte count of $20,000 / \mathrm{mm}^{3}$ (eosinophils, $52 \%$; neutrophils, 47\%; and basophils, 1\%). The absolute eosinophil count was $10,400 / \mathrm{mm}^{3}$, and the platelets were normal. No immature or atypical cells were present in the peripheral blood. Blood biochemistry and bacterial and parasitic studies were normal. ECG, chest X-ray and abdominal ultrasound examination did not reveal any abnormality.

Histological examination of skin biopsy showed a nonspecific picture consisting mainly of a moderate lymphohistiocytic infiltrate in the dermal papillae and around dermal blood vessels, with papillary edema. Bone marrow aspiration revealed a shift to the left of the myeloid series with a moderate increase in the eosinophilic series. Eosinophilic myelocytes, metamyelocytes and stab forms were seen in the aspirate (Figure 1). However, they were of normal morphology. Other series were normal.

Following admission, the patient was started on oral prednisolone $40 \mathrm{mg}$ daily and sodium valproate 500

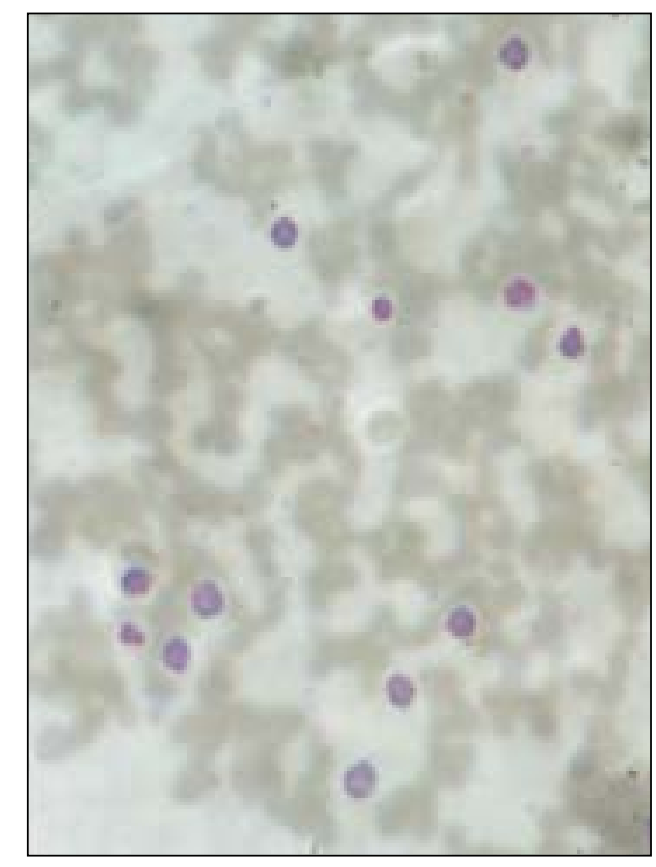

Figure 1: Bone marrow aspirate showing eosinophilic myelocytes, metamyelocytes mg daily. One week after initiation of therapy, the patient's erythema started showing a dusky hue and the scaling decreased markedly. The total leukocyte count and eosinophil count decreased to $9,000 / \mathrm{mm}^{3}$ and $3,150 / \mathrm{mm}^{3}$ respectively after one week and to $6,200 / \mathrm{mm}^{3}$ and $124 / \mathrm{mm}^{3}$ respectively after 2 weeks of steroid therapy. Prednisolone was tapered and stopped over a period of 3 weeks as the erythema and scaling completely subsided, leaving behind post-inflammatory hyperpigmentation. The patient was also treated with emollients, anti-histamines, paracetamol, and a high protein diet.

\section{DISCUSSION}

A leukemoid reaction is defined as a clinical syndrome in which changes are found in the peripheral blood resembling leukemia with the important difference that the former is not an unregulated proliferation of leukocytes but an expression of a bone marrow response to some underlying cause. ${ }^{[5]}$ Eosinophilic leukemoid reactions are often seen in parasitic, allergic, neoplastic, dermatologic, collagen vascular diseases and in drug reactions. ${ }^{|5|}$

In our case, the patient was considered to have an eosinophilic leukemoid reaction because of leukocytosis, a very high absolute eosinophil count $\left(10,400 / \mathrm{mm}^{3}\right)$ and findings of a moderate increase in the eosinophilic series with the presence of eosinophilic myelocytes, metamyelocytes and stab forms of normal morphology on bone marrow aspiration. As leukemoid reactions are always secondary to an underlying cause, they subside on removal of the cause. In our case, patient improved on stopping carbamazepine and after commencement of oral prednisolone therapy.

Several cutaneous and hematological adverse reactions have been observed during carbamazepine therapy. ${ }^{[6]}$ The various cutaneous eruptions caused by carbamazepine include morbilliform eruptions, urticaria, erythroderma, purpura, erythema multiforme, Stevens-Johnson syndrome, toxic epidermal necrolysis, drug induced lupus, and photosensitivity. Various hematological side effects of carbamazepine include leukocytosis, persistent leucopenia, eosinophilia, agranulocytosis, aplastic anemia, and 
thrombocytopenia. The incidence of hematological reactions to carbamazepine has been estimated to range between $1: 10,800$ to $1: 38,000$ per year. ${ }^{[6]}$

Apart from carbamazepine, other drugs that are commonly implicated in causing erythroderma are anticonvulsants like phenytoin and phenobarbitone; antibiotics like penicillins, sulfonamides, and tetracyclines; anti-tuberculous drugs; heavy metals like arsenic, mercurials, and gold; anti-malarial agents; and

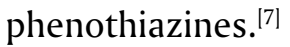

Drug hypersensitivity syndrome (DHS) is characterized by a clinical triad of fever, skin rash, and internal organ involvement. ${ }^{[8]}$ Since its first description in relation to dapsone administration, it has been given several descriptive names such as dapsone syndrome, Kawasaki-like illness, drug reaction with eosinophilia and systemic symptoms (DRESS), graft-versus-host like illness, febrile mucocutaneous syndrome, drug-induced delayed multiorgan hypersensitivity syndrome (DIDMOHS), and mononucleosis-like illness. ${ }^{[8]}$

Common drugs causing drug hypersensitivity syndrome are dapsone, sulfonamides, carbamazepine, phenytoin, phenobarbitone, lamotrigine, allopurinol, azathioprine, minocycline, and the anti-retroviral drugs abacavir and nevirapine. ${ }^{[8]}$ The most recent additions to this list include teicoplanin and amitriptyline. ${ }^{[9,10]}$ This syndrome characteristically occurs on first exposure to the drug and the reaction classically begins 1-8 weeks into drug therapy. ${ }^{[8]}$ The pathogenesis of DHS is not fully understood but it is best categorized as an idiosyncratic reaction. Defective metabolism of the parent compound leading to increased levels of toxic or reactive metabolites is the initial event in the pathogenesis of DHS. The role of these toxic metabolites in an immunologically mediated reaction may be explained by two hypotheses: the hapten hypothesis and the danger hypothesis. ${ }^{[8]}$ According to the hapten hypothesis, reactive drug products bind to tissue macromolecules to form complete immunogens which initiate an immunological process. According to the danger hypothesis, oxidative cell damage caused by reactive drug metabolites leads to release of cytokines that warn the immune system of cellular stress and damage. These signs of danger promote an immune response to eliminate these modified, potentially damaged and dangerous cells. Exogenous precipitating factors for developments of DHS include viruses such as HIV and HHV-6. ${ }^{[11]}$

We have reported this case to highlight the potential of carbamazepine in causing serious dermatological and hematological adverse reactions.

\section{REFERENCES}

1. Brain $C$, MacArdle $B$, Levin S. Idiosyncratic reactions to carbamazepine mimicking viral infections in children. Br Med J 1984;289:354.

2. Yates P, Stockdill G, McIntyre M. Hypersensitivity to carbamazepine presenting as pseudolymphoma. J Clin Pathol 1986;39:1224-8.

3. Lewis IJ, Rosenbloom L. Glandular fever-like syndrome, pulmonary eosinophilia and Asthma associated with carbamazepine. Postgrad Med J 1982;58:100-1.

4. Ray-Chaudhari K, Pye IF, Boggild M. Hypersensitivity to carbamazepine presenting with a leukemoid reaction, eosinophilia erythroderma and renal failure. Neurology 1989;39:436-8.

5. Silver RT. Leukemoid reactions. In: Wyngaarden JB, Lloyd HS, editors. Cecil textbook of Medicine. $19^{\text {th }}$ Ed. Tokyo: WB Saunders; 1992, p. 917-8.

6. Sweetman SC, Ed. Martindale. The complete drug reference. London: Pharmaceutical Press; 2002.

7. Karakayli G, Beckham G, Orengo T, Rosen T. Exfoliative dermatitis. Am Fam Physician 1999;59:625-30.

8. Sullivan JR, Shear NH. The drug hypersensitivity syndrome. What is the pathogenesis? Arch Dermatol 2001;137:357-64.

9. Perrett CM, McBride SR. Teicoplanin induced drug hypersensitivity syndrome. Br Med J 2004;328:1292.

10. Milionis HJ, Skopelitou A, Elisaf MS. Hypersensitivity syndrome caused by amitriptyline administration. Postgrad Med J 2000;76:361-3.

11. Descamps V, Valence A, Edlinger C, Fillet AM, Grossin M, Lebrun-Vignes B, et al. Association of human herpes virus 6 infection with drug reaction with eosinophilia and systemic symptoms. Arch Dermatol 2001;137:301-4. 